\title{
IV GOOD-PRACTICE-EMPFEHLUNGEN
}

\section{Einleitung}

Ein wesentliches Ziel des Projekts COVID-FLUINT lag neben der Akquisition neuer wissenschaftlicher Daten in der handlungsrelevanten Umsetzung der Resultate und in weiterer Folge in der Formulierung konkreter Handlungsempfehlungen.

Um die nach wie vor bestehenden Practice-Defizite auszugleichen und die vielfältigen Potenziale der geflüchteten Menschen besser erschließen zu können, sind gezielte Maßnahmen vonnöten. Bestand zum Zeitpunkt der Datenerhebung im Sommer 2020 noch die berechtigte Hoffnung, dass es mit dem ersten Lockdown in den Monaten MärzApril getan sein könnte, so wurden wir alle im November eines Besseren belehrt und inzwischen haben sich sogar weitere Lockdowns angeschlossen.

Deutlich spiegelten sich in unserem Sample die Komplexität der Rahmenbedingungen und Auswirkungen der COVID-19-Krise sowie die vielfältigen daraus resultierenden integrationspolitischen Konsequenzen wider. Konsens bestand seitens der Betroffenen, Forscher/inn/en und Expert/inn/en darüber, dass es sinnvoll ist, Integrationsbemühungen so weit als möglich weiter zu fördern.

Die Intersektionalität von Pandemie und Flucht berührt eine breite Palette an Politikfeldern und Systemen auf sehr divergente Art und Weise. Die äußerst vielschichtige Thematik der Integration Geflüchteter ist allerdings hinsichtlich der Medienberichterstattung, der politischen Entscheidungsfindungsprozesse, aber auch der institutionellen Maßnahmenkonzipierung sowie im politischen Diskurs infolge der Pandemie stark in den Hintergrund gedrängt worden. Keineswegs verschwunden sind aber nicht selten diskriminierende Zuschreibungen und vorurteilsbehaftete Stereotypisierungen durch Ämter, Behörden Exekutive und in Alltagsinteraktionen, von denen in unseren Interviews seitens der Geflüchteten und der Expert/inn/en berichtet wurde. Gegen diese möchten wir vor allem angesichts der Vulnerabilität der Betroffenen mit einigen unseren Best-Practice-Vorschläge auch Stellung beziehen.

Die zum Zeitpunkt unserer Erhebungen dominierenden Rahmenbedingungen ließen Defizite in den auf unterschiedlichste Aspekte der Integration bezogenen Maßnahmen erkennen. Auf den dringenden Handlungsbedarf haben die befragten Expert/inn/en mit zahlreichen Empfehlungen an das Forscher/innenteam hingewiesen. Bereits in der Zeit vor der COVID-19-Pandemie sahen sich Menschen, die in den letzten Jahren in Österreich Asyl erhielten bzw. darum ansuchten in Bezug auf ihre soziale und Systemintegration extrem herausfordernden und komplexen Aufgaben gegenüber. 
Die gesundheitspolitischen Maßnahmen gegen die Pandemie und ihre vielfältigen Auswirkungen haben in alle zentralen Lebensbereiche eingegriffen und zum Teil völlig neue Hindernisse und Problemkonstellationen geschaffen. Die (mit zeitlich begrenzten Lockerungen) nun bereits seit März 2020 andauernden vielfältigen Beschränkungen des öffentlichen Lebens und somit der sozialen Kontakte wirken sich überaus negativ auf die wichtigen sozialen Netzwerke aus. Diese bieten Hilfe und Unterstützung in wesentlichen Bereichen wie etwa bei der Integration in den Arbeitsmarkt, der Wohnungssuche, bei der Entscheidung über Bildungsverläufe oder der Betreuung von Kindern bzw. älteren Personen. Die Relevanz dieser sozialen Netzwerke wirken auf das gesamte soziale Leben. Wie aus eigenen und internationalen Studien (BERNHARD \& RöHRER 2020; Cheung \& Phillimore 2013; Danzer \& Ulku 2011; Fawcett 1989; Hurlbert et al. 2000; Kohlbacher \& Schiocchet 2017; Kohlbacher 2020a, b; McMichael \& MANDERSON 2004; SMith 2012) in den vergangenen Jahren hervorging, war Social Networking für Viele die wichtigste Strategie auf dem Wege zu einer raschen Integration, zu Sprachlernprozessen und zur Wohnintegration.

Die mit COVID-19 einhergehende wirtschaftliche Krise, die primär jene Branchen erfasst hat, in denen die Respndent/inn/en in der Anfangsphase ihrer Arbeitsmarktintegration besonders oft und leichter Fuß fassen konnten (z.B. Gastronomie, Hotellerie und Fremdenverkehr, Reinigung), erschwert nun den Ersteintritt in den österreichischen Arbeitsmarkt sowie in logischer Folge das weitere Fortkommen. Geflüchtete befinden sich oft erst seit kurzer Zeit in Anstellungsverhältnissen auf dem Arbeitsmarkt, sie sind vermehrt in der Gastronomie und Hotellerie sowie in anderen saisonabhängigen Sektoren tätig und daher viel eher vom Jobverlust betroffen. Die Annahme ist naheliegend, dass das Ankommen und die Integrationsverläufe nun erheblich mehr Zeit in Anspruch nehmen werden als vor dem März 2020.

Im Rahmen der kompilierten Empfehlungen stehen bestimmte Felder im Vordergrund, während andere Bereiche weniger Berücksichtigung finden. Wir haben uns diesbezüglich an unserem empirischen Material orientiert, in dem klare Schwerpunkte erkennbar waren, während andere Probleme seitens der Befragten weit seltener Erwähnung fanden. Im Vordergrund standen die persönliche Gesundheit, die Inanspruchnahme des Gesundheitswesens, die Auswirkungen der Pandemie auf den Zugang zu Bildung, die berufliche Integration und die Bedeutung der NGOs sowie der Organisationen der Communities zur Bewältigung der Krise. Es werden auch die Auswirkungen im Zusammenhang mit dem Deutscherwerb, den Zugang zu weiterführender Schulbildung, berufliche Höherqualifizierung etc. berücksichtigt. Weitere in unterschiedlichen Kontexten angesprochene Aspekte bezogen sich auf Verbesserungen bei der Vernetzung von Institutionen und Organisationen, die Informationsbereitstellung zu COVID-19, Defizite und Strategien in der Informationsdiffusion, Hürden im Zugang zu relevanten Informationen, den Bedarf an Antidiskriminierungsmaßnahmen etc. Es ist zu betonen, dass die Resultate nur eine Momentaufnahme vor dem zeitlichen Hintergrund des Erhebungszeitraumes bilden können, sie beziehen sich auf den ersten Lockdown des 
Jahres 2020 und spiegeln auch eine gewisse Entspannung der Pandemiesituation in den Monaten danach und bis Mitte Oktober 2020 wider.

Darüber hinaus ist festzustellen, dass es weiterer empirischer Analysen zu Entscheidungsprozessen und zur Implementierung von Maßnahmen im internationalen Flüchtlingsregime als Reaktion auf die globale Pandemie bedarf. Dabei sollten verstärkt Non-Governmental-Akteur/inn/e/n, vor allem die Geflüchteten selbst, unterschiedliche Communities und die Zivilgesellschaft, d.h. diverse privat Engagierte, wie etwa Ehrenamtliche, berücksichtigt werden.

Wir verwenden den im wissenschaftlichen Diskurs häufig kritisierten Terminus der „Integration“, obwohl wir uns dessen Problematik und der teils sehr unterschiedlichen Definitionen völlig bewusst sind. „Integration“ und „Inklusion“ sind zwei Begriffe, die im Zusammenhang mit Migration häufig als Auslöser von ideologisch fundierten, kontroversiellen Debatten fungieren. Nicht selten werden sie synonym benutzt, viele Wissenschaftler/innen wie auch Praktiker/innen orten allerdings erhebliche Differenzen. Da es sich hier aber um eine praxisorientierte Kompilation von Handlungsempfehlungen handelt, halten wir die Verwendung eines weit gefassten und kritisch angewendeten Integrationsbegriffs hier für angebracht. 


\section{VULNERABILITÄT}

„Refugeehood is a vulnerability contest” (HowDEN \& KoDALAK 2018) und „,all refugees are vulnerable“ (AGIER 2011, 158) - diese beiden Zitate charakterisieren die generelle und vielschichtige Betroffenheit. In Dokumenten des United Nations High Commissioner for Refugees (UNHCR) wird „,vulnerability“ als "the risk of exposure of refugee households to harm, primarily in relation to protection threats, inability to meet basic needs, limited access to basic services, and food insecurity, and the ability of the population to cope with the consequences of harm" (UNHCR o.J., 1; vgl. auch UNHCR \& IDC 2016) definiert. Konkrete, ausdifferenzierte Kriterienkataloge wurden seitens des UNHCR (o.J.) erstellt und ein "Vulnerability Assessment Framework" (THE Un Refugee AgENCy (2019) sowie ein "Vulnerability Screening Tool” (UNHCR \& IDC 2016) zur Verfügung gestellt.

Gemäß ATAK et al. gelten jene Personen als vulnerable „,... with limited or reduced capacity, power, or control to protect their interests relative to other agents. From a biological or physiological perspective, vulnerability refers to a person's inherent characteristics and to a lack of means to cope without damaging loss“ (ibid. 2018, 2). CHAMBERs $(2006,33)$ konstatiert, dass „Vulnerability here refers to exposure to contingencies and stress, and difficulty in coping with them. Vulnerability has thus two sides: an external side of risks, shocks, and stress to which an individual or household is subject; and an internal side, which is defencelessness, meaning a lack of means to cope without damaging loss. Loss can take many forms becoming or being physically weaker, economically impoverished, socially dependent, humiliated or psychologically harmed.“

Vulnerabilitätskontexte sind nicht fix, sondern wandeln sich im Zeitverlauf sowie in Abhängigkeit von den jeweils gegebenen Rahmenbedingungen (vgl. KOHLBACHER \& SIX-HohEnBALKen 2019, 2020). Unsere Daten belegen, dass die generelle Vulnerabilität von Geflüchteten durch die COVID-bezogenen Maßnahmen erheblich verstärkt wurde. Dies gilt nicht nur für die Angehörigen von Risikogruppen, sondern für Menschen, die flüchten mussten, generell.

Bereits im Erwerbsleben Stehende haben ihre Beschäftigungen verloren, Schüler/ innen, Kursbesucher/innen und Praktikant/inn/en mussten ihre Ausbildungen unterbrechen, Eltern und Kindern wurde die oftmals nicht bewältigbare Aufgabe des Homeschoolings in Wohnungen mit geringen Wohnflächen, ohne IT-Equipment und ohne zusätzliche Hilfestellungen auferlegt.

Weiters wurde der Familiennachzug ausgesetzt. In den Herkunftsländern wurden Krieg, Gewalt und Zusammenbruch von Infrastruktur unvermindert fortgesetzt, auch die Situation in den Transitländern bzw. in Transitlagern hatte sich weiter verschlechterte (siehe Brand von Moria oder Explosion im Hafen von Beirut). Im schlimmsten Fall konnte trotz des positiven Entscheids des Familiennachzuges, dieser nicht reali- 
siert werden und Menschen wurden Opfer des Krieges. Ältere haben zusätzlich zu der Gefährdung infolge ihrer Zugehörigkeit zur Hauptrisikogruppe soziale Netzwerke verloren und leiden an sozialer Isolation.

Während des ersten Lockdowns entstand bei vielen Befragten der Eindruck, dass sie vor allem von der Exekutive als Menschen zweiter Klasse behandelt wurden, und aufgrund xenophob motivierter Vorurteile besonders häufig mit unverhältnismäßig hohen Strafen im Zusammenhang mit behaupteten Verstößen gegen die geltenden COVID-19-Verordnungen belangt wurden. Ihre Vulnerabilität wurde durch diskriminierende Erfahrungen und xenophob motivierte Handlungen seitens mancher staatlicher Organe zusätzlich verstärkt. Ein Thema in den Interviews waren auch österreichische Medien, wobei vor allem Printmedien gemeint waren. Respondent/inn/en erkannten in diesen einen Medienbias, der sich gegen Geflüchtete äußerte, essentialisierend und oft stereotypisierend ausgerichtet war und ihre Vulnerabilität verstärkte. Daher wurde häufig die Forderung nach einem verantwortungsbewussteren Umgang mit der sensiblen Flüchtlingsthematik und persönlichen Daten verbalisiert.

Aus den Interviews ging auch hervor, dass vor allem für Ältere, für die bereits in der Phase vor COVID-19 wenige Angebote vorhanden waren, die Situation verschärft wurde. Ältere repräsentieren weder eine Zielgruppe der Maßnahmen zur Arbeitsmarktintegration noch in zivilgesellschaftlichen Initiativen. In bestehende Angebote für vulnerable Gruppen von NGOs waren sie einbezogen, diese wurden jedoch im Lockdown schlagartig gecancelled bzw. auf digitale Medien umgestellt. Nicht alle kamen damit wirklich zurecht. Für den Zugang zu Social Media waren nicht wenige auf ihre Kinder bzw. Enkel angewiesen, damit waren sie viel stärker als vor der Pandemie von Integrationsangeboten ausgeschlossen. Sprachkurse waren auch zuvor oft nicht auf die Bedürfnisse und speziellen Herausforderungen Älterer ausgerichtet.

\section{Good-Practice-Empfehlungen}

- Geflüchtete als besonders vulnerable Gruppe sollten in künftigen Entscheidungsprozesse über Maßnahmen angehört und wenn möglich einbezogen werden, sodass den speziellen Bedürfnissen mehr Rechnung getragen werden kann.

- Expert/inn/en plädieren für eine stärkere Individualisierung bestehender sowie die Installierung neuer Maßnahmen, um den unterschiedlichen und vielfältigen Bedürfnissen und Fähigkeiten zu entsprechen. Die Rechte, Wünsche und Fähigkeiten der Menschen sollen bei der Formulierung und Gestaltung von Maßnahmen generell stärker berücksichtigt werden. Durch die Berücksichtigung persönlicher Potentiale, durch das Empowerment von Frauen kann die Eigenverantwortung ermöglicht und gestärkt werden. Auch in Pandemiezeiten sollte ein spezifischeres Eingehen auf das jeweilige Individuum und dessen Bedürfnisse zumindest teilweise möglich sein.

- Weiters sollte die Politik auch in herausfordernden Pandemiezeiten nicht den Blick von manifester Diskriminierung und Xenophobie abwenden. Dieser ist nach 
Möglichkeit durch explizite mediale Verurteilung und Schulungen des entsprechenden Personals in adäquater Weise entgegenzusteuern.

- Hervorzuheben ist gerade in herausfordernden Zeiten einer Krise, dass die Schaffung und Gewährleistung von dauerhaften und realistischen Perspektiven von eminenter Wichtigkeit für den individuellen Integrationsverlauf sind. Bei älteren Personen und insbesondere bei Frauen sind die Möglichkeiten infolge von arbeitsmarktabhängigen oder familiären Rahmenbedingungen und nun zusätzlich durch die Pandemie stärker eingeengt.

- Zusätzlich zu diesem Individualansatz erscheint uns aber auch wichtig, einen möglichst ganzheitlichen Blick auf die Anforderungen in den verschiedenen Bereichen der Sozial- und Systemintegration und vor allem auf deren vielfältige Wechselwirkungen zu gewinnen.

- Generell fehlt, wie einige Expert/inn/en anmerkten, breiteres, mit qualitativen Methoden erhobenes empirisches Datenmaterial darüber, wie die Maßnahmen der Regierung sowie die Angebote der NGOs bei den zahlreichen Herkunftsgruppen von Geflüchteten tatsächlich ankommen, wie sie von diesen rezipiert werden, wie es um ihre generelle Compliance steht und inwieweit sie die geforderten Maßnahmen umsetzen können. In diesem Bereich der längerfristigen Wirkungsforschung orten wir ein wichtiges Forschungsdesiderat für die nahe und mittelfristige Zukunft der interdisziplinären sozialwissenschaftlichen Forschung zu vulnerablen Gruppen in Krisenzeiten im Allgemeinen und für Geflüchtete im Speziellen.

- Zweifellos ist ein Mehr an Realitätsbezug hinsichtlich der Erwartungen vonnöten. Geflüchtete können in vielen Aspekten nicht mit den Maßstäben der etablierten Bevölkerung gemessen werden, auch nicht mit den Maßstäben von Arbeitsmigrant/inn/en, die sich bewusst und in geordneten Rahmenbedingungen für Mobilität entschieden haben, um ihre Kompetenzen auf dem Arbeitsmarkt des Ziellandes einzusetzen. Nicht wenige Menschen mit Fluchterfahrungen - diese sind mit dem eigenen unsicheren rechtlichen Status oder dem ihrer Familie konfrontiert - müssen sich erst die im Aufnahmeland umsetzbaren Qualifikationen erwerben und zählen folglich zu den von COVID-19 und den damit einhergehenden Lockdown-Maßnahmen besonders betroffenen Gruppen.

- Von allen Befragten wird im Sinne der Reduktion von Vorurteilen eine Versachlichung teils stigmatisierender Behandlung gefordert. Übergreifende mediale Strategien wären in diesem Zusammenhang als Ziele zu formulieren. Etliche Befragte haben diskriminierende Erfahrungen im Umgang mit der Exekutive machen müssen und als willkürlich erlebte Verwaltungsstrafen auferlegt erhalten. Im Zusammenhang damit wären zusätzliche Antidiskriminierungsmaßnahmen und Schulungen der Mitarbeiter/innen der Exekutive zur Förderung von interkulturellem Verständnis und der Reduktion von Vorurteilen notwendig.

- Aufgrund der sich verändernden demographischen Zusammensetzung der Geflüchteten würden sich spezielle Angebote für Ältere und für chronisch Kranke empfehlen. 


\section{Physische UND PSYChISCHE GeSUNDHEIT}

Wie der Public-Health Experte Martin SPRENGER ${ }^{15}$ bezüglich gesamtgesellschaftlicher Folgen betont, hat sich soziale Ungleichheit durch die Pandemie verschärft. Dies hat Auswirkungen auf die Gesundheit, Erkrankungsrisiken und das Wohlbefinden.

Die Pandemie sowie die darauf bezogenen Maßnahmen haben die Gesundheit und das Wohlbefinden der Geflüchteten stark beeinträchtigt. Um die Auswirkungen von COVID-19 besser im Detail abschätzen zu können, wären Vergleichswerte aus der Zeit vor der Coronakrise notwendig, die aber leider nicht verfügbar sind.

Um genaueren Aufschluss über die Art der gesundheitlichen Belastungen zu erhalten, wurden diese in der vorliegenden Studie abgefragt. Bezogen auf die Grundgesamtheit haben $63 \%$ angegeben, psychische Beschwerden verspürt zu haben, jeweils $19 \%$ hatten als Konsequenz der Pandemie organische bzw. eine Kombination aus organischen und psychischen Problemen. Aufschlussreich ist, dass wesentlich höhere Anteile der Befragten über psychische als über physische Beschwerden klagten, nämlich $63 \%$ der afghanischen und $62 \%$ der syrischen Befragten. Psychische Probleme verschiedenster Art, von Depressionen über Angststörungen bis zu komplexen Retraumatisierungssymptomatiken wurden sowohl von den interviewten Expert/inn/en als auch den Interviewpartner/inne/ $\mathrm{n}$ in den narrativen Interviews als weit verbreitete Corona-Folgewirkungen in allen Altersgruppen beschrieben. Die Akkumulation von psychischen Belastungen hatte in einigen Fällen sogar Re-Traumatisierungseffekte zur Folge. In Extremfällen akkumulierten sich die psychischen Probleme sogar zu einer regelrechten „Coronaphobie“.

Zwei besonders häufig auftretende Stressoren waren der Verlust von Sozialkontakten sowie finanzielle Probleme. Danach folgen mit einigem Abstand die Unklarheit über die Situation von Angehörigen und Probleme im Rahmen der Ausbildung bzw. des Studiums. Weitere Faktoren waren ausbildungsbezogenen Probleme, familiäre Konflikte, die Unklarheit über die Situation von Familienangehörigen, die Kinderbetreuung, sprachliche Barrieren, die Schließung des eigenen Unternehmens und schulische Probleme der Kinder. Einige Befragte haben angesichts steigender Infektionszahlen auch bereits im Spätsommer ihre Angst vor einer zweiten Welle und weiteren Lockdowns bekundet.

\section{Good-Practice-Empfehlungen}

- Die unterschiedlichen Beeinträchtigungen der physischen und vor allem auch der psychischen Gesundheit, wie Depressionen, Angststörungen und Retraumatisierungen, ist als negative Folgewirkung hervorzuheben. Um hohen gesellschaftlichen Folgekosten z.B. auch für andere Integrationsbereiche, wie beispielsweise die berufliche Integration, entgegenzusteuern, ist eine breite

15 Vgl. $<$ https://orf.at/stories/3211006/>. 
Offensive an psychologischer Betreuung, Unterstützung und Therapieangeboten zu empfehlen. Wichtig ist hierbei auch die Zurverfügungstellung von Dolmetscher/inne/n in den Herkunftssprachen und ein ausgewogenes Angebot an Faceto-Face-Betreuung und-Beratung.

- Hinsichtlich der psychologischen Betreuung ist ein höheres Maß an Bewusstsein für die Auswirkungen traumatischer Erlebnisse wichtig. Dieser Aspekt wäre generell in allen Integrationsbereichen stärker zu berücksichtigen. Personen, die schwierige und gewaltvolle Erfahrungen zu verarbeiten haben, sollen die notwendige professionelle Unterstützung zur Verfügung gestellt bekommen, um übliche Alltagssituationen und die generellen und langfristigen Belastungssituationen infolge der COVID-19-Pandemie bestmöglich bewältigen zu können. 


\section{Rolle DES UND KONTAKTE ZUM GESUNDHEITSWESEN UND DESSEN AKTEUR/INN/EN}

Dem hohen Ausmaß an Stress und gesundheitlichen Problemen generell Rechnung tragend wurde auch erhoben, welche Personen bzw. Institutionen zwecks Hilfestellung konsultiert wurden. Am öftesten haben die Befragten Allgemeinmediziner/innen bzw. Fachärzt/inn/e/n mit muttersprachlichen Kompetenzen, Ärzt/inn/e/n aus demselben Herkunftsland kontaktiert. Etwas weniger häufig wandte man sich an eine/n österreichischen praktischen Arzt/Ärztin oder Facharzt/-ärztin. Hervorzuheben ist daher das hohe Maß an Vertrauen, das dem medizinischen Personal mit den jeweiligen muttersprachlichen Kompetenzen entgegengebracht wird. Dies scheint sich nicht primär auf eine Fachkraft aus der engeren eigenen Herkunftscommunity zu beziehen, sondern auch auf Personen, die die jeweiligen Sprachkompetenzen aufweisen. Dabei waren Ärzt/inn/e/n nicht nur Ansprechpartner/innen für medizinische Belange, sondern auch für weitreichende Schwierigkeiten, die im Zuge der Pandemie entstanden sind, und Schlüsselpersonen für unterschiedliche Problemstellungen, wie rechtliche Fragen oder Homeschooling.

\section{Good-Practice-Empfehlungen}

- Das hohe Vertrauen, das in das österreichische Gesundheitssystem gelegt wird, ist hervorzuheben. Dieses steht in einem offensichtlichen Gegensatz zu den qualitativen Mängeln in den Gesundheitswesen in den Herkunfts- und Transitländern, die in dieser Hinsicht häufig stark kritisiert wurden.

- Die Wichtigkeit muttersprachlicher Kompetenz ist offensichtlich ein wesentlicher Faktor, der für das Aufsuchen von bestimmten Ärzt/inn/en spricht. Dies spricht für die Notwendigkeit des Ausbaus der muttersprachlichen Übersetzer/ inne/n und Dolmetscher/inne/n, für Arzt/Ärztin-Patient/inn/en-Gespräche, stationäre Krankenhausaufenthalte, Erklärungen von ärztlichen Befunden und der Medikation. Angeraten wird die intensivere Nutzung von bereits seit 2013 (damals noch im Pilotversuch) installierten digitalen Möglichkeiten der Involvierung von Video- oder Telefondolmetscher/innendiensten. Dies spricht auch für den Ausbau von „,Distance translating “-Einrichtungen.

- Während des ersten Lockdowns bedeutsam war der communitybasierte Aufbau von Netzwerken muttersprachlich versierter Ärzt/inn/e/n, um als erste Anlaufstelle zu fungieren. Im engen Konnex zu medial immer wieder geäußerten Klagen über den Mangel an Fachkräften im Gesundheitswesen steht die schnellere Nostrifikation von im Ausland abgeschlossenen Medizinstudien und Krankenpflegeausbildungen. Gerade in Syrien entspricht das Niveau der Ausbildung hohen Standards und syrische Ärzt/inn/e/n könnten zum Beispiel den Mangel 
an Mediziner/inne/n kompensieren helfen. Die neu geschaffene höherwertige Pflegeausbildung böte auch eine Zukunftschance für viele Qualifizierte, die in ihrem bislang ausgeübten Beruf aufgrund der pandemiebedingten Wirtschaftskrise derzeit sehr schlechte Chancen auf dem Arbeitsmarkt vorfinden.

- Expert/inn/en berichteten auch, dass sie vermehrt Instant-Messaging-Dienste wie WhatsApp nutzten, um mit Patient/inn/en zu kommunizieren. Diese Dienste waren niederschwelliger, als schriftliche Kommunikation per E-Mail oder Brief und erlaubten zudem Sprachnachrichten aufzunehmen und Befunde bzw. sichtbare Gebrechen abzufotografieren. Da die Kommunikation und der Austausch sensibler Daten über Instant-Messaging-Dienste jedoch rechtlich in eine Grauzone fallen, könnte eine Ausarbeitung von Empfehlungen hinsichtlich der Nutzung für Ärzt/inn/e/n eine Orientierung bieten. 


\section{INFORMATIONSBESCHAFFUNG UND -DISTRIBUTION}

Infolge der großen Menge und sich rasch ändernden Inhalte der Nachrichten insgesamt sowie der Berichterstattung zu den Maßnahmen fühlten sich viele Geflüchtete und NGOs genauso wie in Österreich geborene oder lange hier ansässige Personen in der Frühphase des Lockdowns eindeutig überfordert. Eine besondere Herausforderung bestand in den kurzen „Halbwertszeiten“ der meisten Informationen und auch der geltenden Maßnahmen, die sich sehr rasch änderten und mitunter auch nicht klar formuliert bzw. kommuniziert wurden. Die damit verbundenen Herausforderungen waren gerade für Personen mit oft geringen Deutschkenntnissen noch erheblich größer. Die Sprachbarriere(n) in der Informationsvermittlung und -rezeption waren eine extreme Herausforderung für viele. Selbst Mediziner/innen hatten in diesem Feld mit Schwierigkeiten in der Informationsbeschaffung zu kämpfen. Faktum war, dass auch die offiziell eingerichteten staatlichen Hotlines kein mehrsprachiges Personal zu Verfügung hatten und ausreichende Informationen oder Beratung nicht einmal auf Englisch angeboten wurden.

Die NGOs sowie die Vereine der Communities kamen dem großen Informationsbedarf möglichst rasch entgegen, indem sie spezifische Hotlines zu bestimmten Problemen (z.B. physische/psychische Gesundheit, Homeschooling) installierten. Auch unter Verwendung der sozialen Medien, haben sich WhatsApp-, Facebook- und ViberGruppen gebildet, um Informationen weiterzugeben. Vereine haben sich so über das gesamte Bundesgebiet vernetzt. Es gab eine Reihe von Initiativen in den arabischen Communities und auch Einzelpersonen, die sich auf die Übersetzungen von österreichischen Medien fokussiert haben. In den Befragungen war daher das Vertrauen in österreichische Medien relativ hoch und stand auf den ersten Blick in einem Widerspruch zu der eigenen Einschätzung von unzureichenden Sprachkenntnissen.

Ärzt/inn/e/ninitiativen, die Hotlines gründeten, oder auch bereits sehr etablierte Hilfsvereine haben über soziale Medien relativ rasch und effizient unterschiedliche Informationskanäle eröffnet. Auch sind erste Projekte entstanden, in welchen diese Kompetenzen in/aus den Communities von Institutionen des Gesundheitswesens aufgenommen, übernommen oder adaptiert wurden.

Mit längerer Dauer der Pandemie und des Lockdowns verblieb der Informationsbedarf auf einem zwar hohen Niveau, viele Menschen lernten aber zunehmend eigenständig mit unterschiedlichen Informationsquellen umzugehen, diese parallel zu rezipieren und sich kritisch mit deren Inhalten auseinanderzusetzen. Dies wie auch die Aneignung von IT-Kompetenzen kann als ein positiver Nebeneffekt dieser herausfordernden Zeit für viele Menschen bewertet werden. Der Erwerb einer höheren Medienkompetenz, insbesondere von Sicherheit und Routine im Umgang mit digitalen Medien, die kritische Reflexion der verfügbaren Meldungen und Inhalte und die Auseinandersetzung mit den Auswirkungen der Krise für das persönliche Leben in den unterschiedlichen Sphären der sozialen und der Systemintegration zählten dazu. 
Da auch die sogenannten ,gatekeepers“ im System, die Betreuungseinrichtungen im Fluchtbereich, während des ersten Lockdowns geschlossen waren, kam es gerade beim Zusammentreffen von mehreren Problembereichen zu heiklen Situationen. Menschen, die mit dem Gesundheits-, Rechts- und Sozialwesen in Österreich noch nicht ausreichend vertraut waren, wussten nicht, wo sie entsprechend qualifizierte und verlässliche Hilfe finden konnten. Auch die Ärzt/inn/e/n der Hotline, die telemedizinische Hilfestellungen anboten, waren oft überfragt, wenn es beispielsweise um medizinische Spezialfälle ging. Vor allem war auch die Intersektionalität von medizinischen und rechtlichen Fragen in manchen Krisenfällen schwierig und erzeugte bei den Betroffenen vielfach Angst und Unsicherheit.

Auch wenn manche der Befragten schon sehr gute Deutschkenntnisse aufwiesen, meinten Expert/inn/en dass gerade bei spezifischen medizinischen oder rechtlichen Angelegenheiten zusätzlich die Vermittlung in der Muttersprache vorhanden sein sollte, um schwerwiegenden Folgewirkungen entgegenzuwirken. Gerade in Ausnahmesituationen sollte Unklarheiten aufgrund von Sprachbarrieren möglichst entgegengewirkt werden.

Medizinische Expert/inn/en haben darauf verwiesen, dass aufgrund der ethnischen und kulturellen Diversität in Österreich solche Informationsdefizite nicht alleine Geflüchtete betreffen, sondern auch Angestellte bei internationalen Organisationen, Betrieben oder Expats. Die Ausweitung der Übersetzung von Informationsmaterial würde daher auch der internationalen Ausrichtung in Gesellschaft und Wirtschaft Rechnung tragen. Auch in dieser Hinsicht müssen Entscheidungsträger/innen ihre Kommunikationsstrategien und ihre Wortwahl überdenken und in offiziellen Ansprachen auch alle in Österreich lebenden Personen entsprechend einbeziehen.

Informations- und Kontaktprobleme ergaben sich auch im Zugang zu Behörden wie dem AMS oder der MA 40 hinsichtlich des Bezuges von Arbeitslosengeld sowie der bedarfsorientierten Mindestsicherung. Damit waren Personen mit Bildungsdefiziten, mit geringeren Deutschkenntnissen oder fehlendem technischem Equipment überfordert. Zudem ergaben sich durch mangelndes Schnittstellenmanagement und verlangsamte bürokratische Prozesse gerade dann Probleme, wenn Personen in andere Bundesländer übersiedelten oder ihren rechtlichen Status änderten.

Social Media spielten eine ganz wichtige Rolle in der Informationsdiffusion und es war eine Reihe von Einzelinitiativen vorhanden, die über soziale Medien spezifische Informationen gepostet haben. Diese privaten Seiten in den sozialen Medien wurden seitens der Expert/inn/en aber nicht nur positiv bewertet, da die Informationen nicht immer korrekt waren und man diesen weniger Vertrauen entgegenbringen konnte. Eine Expertin meinte auch, dass nicht alle Initiativen in den sozialen Medien adäquat sind, da sie in dem einen oder anderen Bereich Persönlichkeitsrechte der Betroffenen nicht ernst genug nahmen, so z.B. vertrauliche Informationen über Patient/inn/en im Internet auftauchten und bestimmte medizinische oder verwandte Fragen diskutiert oder politische Inhalte transportiert wurden. 
Dass das Misstrauen gegenüber amtlichen Informationen nicht immer ganz ungerechtfertigt war, bekunden sowohl Geflüchtete als auch Expert/inn/en, denn leider wurden sogar von einzelnen offiziellen Stellen nur bedingt richtige oder sogar falsche Informationen verbreitet, was seitens der Communities sehr emotional kritisiert wurde. Dies betraf Informationen z.B. in Einrichtungen der Asylbetreuung, aber auch seitens des ÖIF. Von diesen wurden relativ zeitnah Übersetzungen der von der Regierung gesetzten Maßnahmen in zahlreichen Migrant/inn/ensprachen zur Verfügung gestellt. Allerdings wurde seitens der NGOs sowie der Betroffenen mit Recht kritisiert, dass diese fehlerhaft gewesen oder nur auszugsweise wiedergegeben waren.

Eine breite Palette von geäußerten Desideraten sowohl von Expert/inn/en als auch Geflüchteten bezog sich auf bestehende Informationsdefizite und den Bedarf an Informationsangeboten jeglicher Form in den verschiedensten auf COVID-19 bezogenen Herausforderungen. Hier stellt sich als generelle Frage, wie man den Transfer und die Kommunikation des nun bereits viel reichlicher als zum Zeitpunkt des Beginns der Pandemie vorhandenen Informationsmaterials an die Rezipient/inn/en bewerkstelligen kann. Weiters mitzubedenken sind jene Personen, die aus bildungsfernen Schichten stammten und für die die orale Informationsvermittlung bis dato noch immer ein höheren Stellenwert hat. Manche Communityorganisationen haben hier auch BestPractice-Maßnahmen entwickelt und beispielsweise mittels Videos auf der Homepage laufend Informationen vermittelt.

\section{Good-Practice-Empfehlungen}

- Ein wichtiger Lerneffekt aus der ersten Lockdown-Phase sollte sein, dass die Informationsstrategien systematisch verbessert werden und dass die Transparenz sowie Verlässlichkeit der gebotenen Informationen einen extrem relevanten Aspekt darstellen.

- Die Wichtigkeit eines möglichst breiten und diversifizierten Informationsangebots, die Informationsdiffusion durch Verstärkung von Medienarbeit sowie die Verbesserung des Informations- und Wissenstransfers zwischen bereits bestehenden Systemen und Praktiken ist hervorzuheben. Wir schlagen generell vor, zusätzlich zum schriftlichen Informationsmaterial im Internet weiteres zum Einsatz zu bringen, z.B. YouTube-Filme oder diverses Videomaterial.

- Die Organisationen der Communities haben während und auch noch nach dem Lockdown Beachtliches im Sinne der Information und Unterstützung der Menschen in den Communities geleistet. Die Repräsentant/inn/en der Vereine standen als Ansprechpersonen und für Hilfestellungen jeglicher Art zur Verfügung und haben auf diese Weise ausschließlich ehrenamtlich das beträchtliche Manko an Information und Unterstützung von staatlicher und kommunaler Seite ausgeglichen. Dieser immens wichtigen Rolle Rechnung tragend muss für eine Einbeziehung der Organisationen der verschiedenen Communities in Diskussionen um 
künftige Maßnahmen sowie für entsprechende finanzielle Unterstützung ihrer Aktivitäten plädiert werden.

- Wie unsere Befragung ergeben hat, wurde dem ORF sowie österreichischen Printmedien ein hohes Ausmaß an Vertrauen entgegengebracht. Geringer war das Vertrauen in die Internetseiten von österreichischen NGOs, Institutionen und Vereinen. Einigen Vereinen der Communities vertrauten viele Befragte mehr.

- Besonders hinsichtlich der Sprachbarrieren orten wir auf Basis der Resultate unserer Erhebungen ein ganz essentielles Manko, dem künftig unbedingt entgegengesteuert werden muss, indem die Informationen in möglichst vielen Sprachen zur Verfügung gestellt werden. Diese sollten gründlich auf etwaige Fehlerhaftigkeit (auch Übersetzungsfehler) hin kontrolliert werden Es ist daher dringend anzuraten solche wichtigen Informationen künftig durch mehrere Personen mit Native Language Competence einem gründlichen Check hinsichtlich der Inhalte zu unterziehen.

- Wichtig ist weiters, dass gut qualifiziertes mehr-bzw. fremdsprachiges Personal für die wichtigen Hotlines akquiriert wird.

- Wir plädieren für gebündelte und diversifizierte Informationsangebote für die jeweiligen durch COVID-19 hervorgerufenen, spezifischen Problemkonstellationen sowie für die Ausweitung des spezifischen Informationsangebots für Frauen.

- Kritisiert wurde von Expert/inn/en und Geflüchteten, dass es ein Überangebot an Informationen in den sozialen Medien gab, welche von privaten Übersetzer/ inne/n zusammengestellt wurden. Neben einer Bündelung wäre eine stärkere Zusammenarbeit mit vereinzelten Gatekeepern erstrebenswert. Diese können entweder Sprecher/inn/en oder Repräsentant/inn/en von Organisationen sein, aber auch Moderator/inn/en von besonders relevanten Social-Media-Kanälen. Neben der Anerkennung ihrer bisherigen Eigeninitiativen in der Informationsdiffusion können durch gezielte Schulungen dieser Schlüsselpersonen zudem Kompetenzen im Bereich der Moderation vermittelt werden und dem Umlauf von falschen Informationen entegegengewirkt werden. 


\section{SOZIALE NETZWERKE UND DEREN INTEGRATIONS- RELEVANZ}

Social Networks leisten für den Großteil der Befragten unverzichtbare Hilfestellungen im komplexen Prozess der Integration in Wien. Mit der Flucht geht in der Regel ein weitgehender bzw. völliger Verlust des „social capital“ (PuTNAM 1993, $1995)$ einher. Soziale Kontakte mit Angehörigen im Herkunftsland können dank moderner digitaler Infrastruktur zwar aufrechterhalten werden, schwächen sich aber ab und können vor allem bei der Integration kaum hilfreich sein. Daher kommt der Etablierung sozialer Kontakte mit Menschen (ob mit oder ohne Migrationsgeschichte) im Aufnahmekontext eine eminente Bedeutung zu. Das Knüpfen und die langfristige Aufrechterhaltung sozialer Netzwerke erfordern jedoch einen erheblichen Input an Zeit und vor allem an Kontaktmöglichkeiten. Diese Opportunity Structures wurden infolge COVID-19 zeitweilig fast gänzlich unterbrochen bzw. wurden sie zum Befragungszeitpunkt August/September 2020 erst mühsam wieder reaktiviert. Es sind aber gerade die „Weak ties“ (GranovetTer 1973; 1995; Rose et al. 1998), die für Viele von besonderem Wert sind und ihnen Unterstützung bieten, wo eine gewisse Vertrautheit mit und das Wissen über die institutionellen und kulturellen Regelhaftigkeiten und Normen des Aufnahmelandes von Vorteil sind, allen voran die Suche nach einer Beschäftigung oder Wohnung, die Kompilation von Bewerbungsunterlagen etc. Ehrenamtliche, Mitarbeiter/ innen von NGOs, Nachbar/inne/n, Eltern von Schulfreund/inn/en der Kinder, sowie andere Mitglieder in den Vereinen, in welchen Geflüchtete tätig sind, aber vielfach auch Deutschtrainer/innen können auf der Basis von „,weak ties“ wichtige Hilfestellungen bieten.

Studien (Punz \& Schwarzbauer 2020; Bergeron \& Potter 2006) zeigten darüber hinaus auch die besondere Bedeutung von sogenannten „strong ties“, also besonders engen sozialen Kontakten zu Einheimischen wie Lehrer/inne/n, ehrenamtlich tätigen Personen oder in Vereinen Aktiven. Diese Kontakte leisten weitreichende instrumentelle, soziale und emotionale Hilfestellungen und tragen ganz wesentlich dazu bei, sich in Österreich heimisch zu fühlen.

Wie auch die Daten der vorliegenden Untersuchung zeigen, waren für einen Teil der aus Syrien und Afghanistan stammenden Befragten, die von österreichischen Freund/inn/en/Bekannten eingeholten Informationen zu COVID-19 allgemein sowie den Maßnahmen der Regierung im Besonderen von großer Wichtigkeit. Allerdings hat nur ein Teil der Respondent/inn/en diese Kontakte im Kontext von COVID-19 für sich als sehr relevant beurteilt, nicht allen ist es gelungen, sich in den ersten Jahren ihres Aufenthaltes auf diese Weise in Österreich sozial zu verankern.

Vor allem die Interviews mit den Expert/inn/en aus den NGOS haben gezeigt, dass die Unterstützungsnetzwerke unter den Normen des „Social Distancing“ sowie den ökonomisch-finanziellen Einschränkungen leiden. Kontakt-Cafés und alle Initia- 
tiven, die auf die Förderung des Socializing orientiert waren, mussten quasi über Nacht schließen, auch aufsuchende Sozialarbeit der NGOs und das vielfältige Engagement der Ehrenamtlichen musste infolge der Infektionsprävention eingestellt werden. Dies galt ebenso für die professionelle Beratung und Unterstützung beispielsweise in Form psychologischer Betreuung. Zahlreiche vor dem Lockdown bestehende Freizeitangebote der NGOs mussten ebenfalls eingestellt werden, einige aber keineswegs alle wurden in den Sommermonaten nach und nach wieder aufgenommen. NGO-Expert/ inn/en bekundeten, dass dadurch viele zuvor betreute Personen mit Fluchthintergrund förmlich „weggebrochen“ und für die NGOs nicht mehr erreichbar waren.

Die Maßnahmen zur Eindämmung der COVID-19-Pandemie wirkten sich auf all diese Angebote der Begegnung, sozialen Interaktion, sozialen bzw. psychologischen Unterstützung aus. Damit sind vielfach die für geflüchtete Menschen überaus wichtigen „weak ties“ zum Teil nachhaltig verlorengegangen. Viele der Ehrenamtlichen zählen aufgrund ihres oft bereits fortgeschrittenen Alters und Vorerkrankungen zu den COVID-19-Risikogruppen, sie haben ihr Engagement und ihre Aktivitäten im Erhebungszeitraum nicht wiederaufnehmen können. Ein wenig anders gestaltete sich die Situation hinsichtlich der professionellen Akteur/inn/e/n in den NGOs. Sie haben ihr Beratungs- und Betreuungsangebot relativ rasch auf digitale Medien umstellen können bzw. Beratung auch telefonisch angeboten. Weiters waren sie auch proaktiv tätig und versuchten über regelmäßige Telefonanrufe die Kontakte zu halten. Damit konnte der Ausfall zwar einigermaßen kompensiert, jedoch nicht gänzlich ersetzt werden.

„Strong ties“ zu Familienangehörigen oder Freund/inn/en aus den eigenen Communities haben sich den pandemiebedingten Beschränkungen gegenüber hingegen als robuster erwiesen, da sie auf engen und tragfähigen wechselseitigen Bindungen basieren. Die Menschen kennen einander gut und hatten bereits vor dem Lockdown regelmäßigen telefonischen oder Chat-Kontakt. Die Beschaffung von Informationen über COVID-19 und das Vertrauen in den Wahrheitsgehalt derselben über ,strong ties“ wurden daher in der Befragung häufig genannt.

Eines zeigt sich ganz deutlich: Dass nämlich die sozialen Auswirkungen der Maßnahmen zur Eindämmung der Infektionen grundlegend und nachhaltig in die Unterstützungsnetzwerke jener eingreifen, die besonders stark auf professionelle und ehrenamtliche Hilfestellungen angewiesen sind. Dies trifft vor allem auf die sozial isolierteren Personen, jene mit Bildungsmängeln und rudimentären Deutschkenntnissen, auch auf die kleinere Gruppe der älteren Personen, für die die Arbeitsmarktintegration besonders schwierig ist, zu. Es handelt sich damit um einen Personenkreis, der ohnehin besonders vulnerabel ist und dessen Vulnerabilität durch die Pandemie noch zusätzlich verstärkt wird.

Besonders nachteilig war der Wegfall dieser Betreuungsangebote auch für Kinder, die über Vereine zusätzlichen Sprach- oder Nachhilfeunterricht, eine Nachmittagsbetreuung oder Freizeitgestaltung erhielten. Social Media konnten nicht in Ansätzen den Wegfall dieser Angebote wettmachen. 
Sehr viele Befragte haben auch angegeben, dass ein wesentlicher psychischer Stressfaktor die Sorge um Familienangehörige, die noch im Herkunftsland leben, war. Mit der starken Verbreitung des Coronavirus in Afghanistan, dem Iran, aber auch in Syrien und der Türkei stieg auch der psychische Stress in transnationalen Familienkonstellationen an. Digitale Medien erlaubten zwar den Kontakt, die Geflüchteten waren über die Probleme der Familienangehörigen und Freund/inn/e/n in den Herkunftsländern informiert, wussten wohl über die mangelhaften medizinischen Leistungen Bescheid, aber konnten kaum jemanden aktiv unterstützen.

\section{Good-Practice-Empfehlungen}

- Da der Wegfall der wichtigen unterstützenden Netzwerke nicht völlig verhindert werden kann, ist soweit als möglich für deren Kompensation zu sorgen. Digitale Angebote können hier nur einen Teil des Ausfalles abdecken, da diese nicht für alle gleichermaßen zugänglich sind und es ihnen vor allem an der persönlichen Interaktion und sozialen Nähe fehlt.

- Für die Zeit nach dem derzeitigen Lockdown kann daher dringend empfohlen werden, die breite Palette der Beratungs- und Hilfsangebote möglichst rasch wieder hochzufahren, natürlich unter Berücksichtigung und strikter Einhaltung aller möglichen Maßnahmen der Infektionsprävention.

- Ehrenamtlichen kommt eine tragende Rolle zu. Dies hat sich vor allem in der Phase der Fluchtbewegung von 2015/16 gezeigt. Von Expert/inn/enseite hervorgehoben wurde die immense Wichtigkeit von Ehrenamtlichen und Mentor/inn/ en bzw. Buddies als Informant/inn/en und Begleiter/innen in allen integrationsrelevanten Bereichen, vor allem aber im Feld der sozialen Integration sowie der Übersetzung und Umsetzung der pandemiebezogenen Maßnahmen.

- Zum Zwecke der Aufrechterhaltung der Motivation erwarten sich viele Ehrenamtliche auch eine Anerkennung für ihr Engagement. Mögliche Überforderungen der muttersprachlichen Mitarbeiter/innen (Ehrenamtlichen) sollten so gering wie möglich gehalten werden. Diese Kategorie von Mitarbeiter/inne/n ist nicht automatisch Expert/inn/en gleichzusetzen. Viele verfügen über keine fachspezifische Ausbildung, ihre Überforderung war in der Pandemie geradezu vorprogrammiert. Als Angehörige der Communities befanden sie sich darüber hinaus in einem Zwiespalt, wenn sie unangenehme maßnahmeninduzierte institutionelle bzw. bürokratische Entscheidungen mitzutragen gezwungen waren oder um Intervention und Hilfe z.B. bei Verwaltungsstrafen gebeten wurden.

- Am schwierigsten ist die Kompensation des wichtigen Engagements der Ehrenamtlichen. Realistischerweise ist zu erwarten, dass ein analoges Level an ehrenamtlicher Tätigkeit im Bereich der Unterstützung von Geflüchteten erst wieder nach der COVID-19-Krise mit Sicherheit aber erst nach möglichst flächendeckenden Impfmaßnahmen erreicht werden wird können. 


\section{7 ÖKONOMISCH-FINANZIELLE AUSWIRKUNGEN UND ARBEITSMARKTINTEGRATION}

Arbeitsmarktbezogene Fragen waren ebenfalls ein in unserer Erhebung berücksichtigter Aspekt. Politisch und ökonomisch nimmt die Arbeitsmarktintegration eine besondere Stellung ein - nicht nur aus Sicht des Aufnahmelandes, sondern auch aus Sicht der Geflüchteten selbst (vgl. BUbER-EnNSER et al. 2016; KOHBACHER et al. 2020). Viel wurde zur Arbeitsmarktintegration der seit 2015 in der EU aufgenommenen Personen publiziert (vgl. z.B. BERNHARD \& RÖHRER 2020; BRÜCKER, HAUPTMANN et al. 2020; BRÜCKER, GUNDACKer et al. 2020). Der Einstieg in den Arbeitsmarkt ist in der Anfangszeit vor allem durch Ausbildungsdefizite sowie unzureichende Deutschkenntnisse erschwert. Vorhandene Qualifikationen können oft nicht (voll) anerkannt werden.

Ein Teil der Befragten verfolgt mittel- und langfristige Berufsziele; sie befanden sich zum Zeitpunkt des Lockdowns in ersten Anstellungen oder in Ausbildungsverhältnissen, wollten Schulabschlüsse nachholen oder sich auf ein Studium konzentrieren. Die Unterbrechung dieser Pläne und zahlreicher bereits laufender Ausbildungen wurde von vielen Befragten als besonders einschneidende Auswirkung der Pandemie erlebt, die sie in ihren Integrationsbemühungen gehemmt oder zurückgeworfen hat.

Andere haben auch aus pragmatischen Gründen einen raschen Arbeitsmarkteinstieg ohne berufliche Vorbildung realisiert, über Neben- und Gelegenheitsjobs, Hilfstätigkeiten in diversen Branchen oder als Praktikant/inn/en, um so rasch als möglich Erfahrungen auf dem österreichischen Arbeitsmarkt zu sammeln, ein Einkommen für sich und die Familie zu erwirtschaften, Geldüberweisungen an die Angehörigen im Herkunftsland tätigen zu können oder so bald als möglich einen Anspruch für die Erlangung der Staatsbürgerschaft zu erwerben. Bis vor dem Lockdown war dies infolge der günstigen Beschäftigungs- und Wirtschaftslage und konstanter Arbeitskräftenachfrage in Gastronomie und Tourismusbetrieben relativ einfach möglich.

Die aktuellen wirtschaftlichen Entwicklungen infolge COVID-19 haben eine gänzlich veränderte Situation geschaffen. Viele Befragte haben ihre Beschäftigung verloren, etliche befanden sich zum Erhebungszeitpunkt noch in Kurzarbeit, wer nach dem Lockdown eine Arbeit suchte, wurde kaum fündig, denn gerade Geflüchtete sind in hohem Ausmaß in Bereichen beschäftigt, die nicht zu den krisensicheren Segmenten des Arbeitsmarktes zählen. Viele waren in der Folge von den Bezügen von Leistungen des AMS oder der MA 40 (Sozialhilfe/bedarfsorientierte Mindestsicherung) abhängig. Die Inanspruchnahme privater finanzieller Unterstützung (Verwandte, soziale Netzwerke), um nicht wieder in die Mindestsicherungsschiene zu fallen, war für viele eine kurzfristige Möglichkeit den Folgen der Arbeitslosigkeit (v.a. Wohnungsverlust) entgegenzuwirken. Es gibt Spar-und Kreditkollektive, sogenannte jam 'iyāt (arab.), an denen mehrere Personen beteiligt sind. Dies ist eine Form eines inoffiziellen Sparfonds, in den man regelmäßig einzahlt. Abwechselnd geht der Fonds an jemand anderen, der ihn gerade benötigt, um beispielsweise die Kaution für eine Wohnung zahlen zu können. 
Das Zusammenwirken zahlreicher Faktoren im Kontext der Pandemie und ihrer Bekämpfung hat die ohnehin bestehenden tiefgehenden Brüche auf dem österreichischen Arbeitsmarkt zwischen relativ krisensicheren und stark konjunkturabhängigen Segmenten weiter verstärkt. Dies führte in der Folge zu tiefen und nachhaltigen Brüchen in den individuellen Erwerbsbiografien. Die meisten finden nun deutlich reduzierte Chancen für die Akkumulation beruflicher Erfahrungen auf dem österreichischen Arbeitsmarkt vor, sie können kein Einkommen aus Erwerbsarbeit erzielen, sondern sie und ihre Familien sind von staatlichen Transferleistungen abhängig geworden. Dass damit auch erhebliche finanzielle Einbußen einhergehen, erübrigt sich hinzuzufügen. Damit assoziiert ist eine Akkumulation von psychischen Belastungsfaktoren und Stressoren, die durch die Unsicherheit des Asylverfahrens (viele Afghan/inn/en erhalten nur befristeten subsidiären Schutz), vergebliche Bemühungen um neue Jobs, Diskriminierungserfahrungen auf dem Arbeits- und Wohnungsmarkt charakterisiert sind.

\section{Good-Practice-Empfehlungen}

- Trotz der Abhängigkeit von der schwer prognostizierbaren gesamtwirtschaftlichen Entwicklung sowie jener in den Branchen, in denen Geflüchtete primär Beschäftigung finden, sind gezielte Fördermaßnahmen für die (Re-)integration in den Arbeitsmarkt unabdingbar. Der Arbeitsmarkt repräsentiert den Schlüsselbereich der Integration und der ökonomischen Unabhängigkeit. Es empfehlen sich Bündelungen von Maßnahmen, die auch möglichst schnell nach Ende der Krise installiert werden sollten. Der Zeitfaktor spielt hier eine erhebliche Rolle, da ansonsten bereits angeeignete Kompetenzen wieder verloren gehen könnten und die Gefahr wächst, größere Zahlen von Menschen mit Fluchthintergrund langfristig nicht mehr in den Arbeitsmarkt integrieren zu können. 


\section{BiLdung: HomeschoOling UNd ERLERNEN DER DEUTSCHEN SPRACHE}

Die Betreuung der Kinder im Homeschooling war durchwegs ein erheblicher Stressfaktor für die meisten Familien und hat damit auch das ganze Familienleben bestimmt. Der Wegfall der schulischen Betreuung wurde auch von Seiten der Expert/ inn/en der NGOS als sehr belastend für ihre Klient/inn/en charakterisiert und mit zahlreichen sogar täglich wechselnden Herausforderungen verbunden. Die Expert/inn/ en klassifizierten das Homeschooling somit als eines der größten Problemfelder des Lockdowns, da dies aufgrund der unterschiedlichen Ressourcen eine Schere zwischen Kindern aus ökonomisch bessergestellten und IT-mäßig gut ausgestatteten Haushalten und solchen mit mangelnder Ausstattung öffnete. Kinder mit ohnehin bereits vorhandenen Lernrückständen und -schwierigkeiten oder jene, die gerade eingeschult wurden oder einen Schulwechsel hatten, wurden damit noch weiter zurückgeworfen. Viele Instruktionen seitens der Schulen bzw. Lehrkräfte waren unklar, widersprüchlich und stellten die Befragten angesichts mangelnder Deutschkompetenzen vor fast unlösbare Hindernisse. Selbst wenn Lehrkräfte zusätzliche Unterstützungsleistungen boten, gaben Befragte mit akademischem Bildungsniveau diesbezüglich durchwegs an, Probleme gehabt zu haben, da sich die Lehrpläne und Bildungssysteme in den Herkunftsländern Syrien und Afghanistan von jenen in Österreich grundlegend unterscheiden.

Für die NGO-Mitarbeiter/innen sowie die afghanischen Vereine resultierten daraus völlig neue Herausforderungen, auf die rasch reagiert werden musste. Eigentlich waren nur die wenigsten der Befragten vor dem Lockdown in den Bereich der Lernunterstützung involviert, obwohl einige NGOs solche Angebote natürlich offerierten. Es mussten quasi über Nacht Hilfestellungen im Zugang zu Lernmaterialien geschaffen werden, verschiedenartigste Defizite der digitalen Ausstattung in den Haushalten sowie hinsichtlich deren Umgang mit IT-Infrastrukturen kompensiert werden, den Eltern und Kindern Hilfe in der Bewältigung der Hausübungen zur Verfügung gestellt sowie sprachliche Defizite so gut als möglich ausgeglichen werden etc. Auch NGO-Mitarbeiter/ innen, die im Bereich psychologischer Betreuung tätig waren, wurden mit zahlreichen Ersuchen um Hilfe in Homeschooling-Agenden seitens ihrer Klient/inn/en konfrontiert. In der Phase nach dem Lockdown und relativer Normalität während des Sommers haben manche NGOs zum Zweck der Kompensation von Lerndefiziten zusätzliche Förderkurse für Kinder mit Migrations- bzw. Fluchterfahrungen angeboten.

Ein weiterer wichtiger Aspekt der Bildung ist das Erlernen der deutschen Sprache. Das System der Sprachvermittlung orientiert sich an einer Abfolge von aufeinander aufbauenden Kursen vom Basis-Level (A1-Niveau) bis zum Muttersprachler/in-Level (C2-Niveau). Außer bei besonderer Sprachbegabung dauert es in der Regel Jahre, bis die Sprachkenntnisse für eine höhere Berufsausbildung oder ein Studium ausreichen.

Aufgrund der Maßnahmen zur Eindämmung der Corona-Pandemie wurden Sprachkurse verschoben, ausschließlich nur mehr virtuell abgehalten oder ganz abge- 
sagt. Wartezeiten auf verschobene Sprachkurse wurden von Vielen als verlorene Zeit empfunden und wirkten demotivierend. Viele äußerten die begründete Befürchtung das bereits Erlernte mangels praktischer Übung wieder zu vergessen. Die Einschränkungen der Kontaktmöglichkeiten zu Einheimischen in der Schule, aber auch für Erwachsene generell haben sich negativ auf das Erlernen und die Praxis der deutschen Sprache im Alltag ausgewirkt. Wie CHISWICK et al. (2004) sowie van TuBERGEN (2010) nachgewiesen haben, sind Sozialkontakte der Schlüssel für den Erwerb einer Zweitsprache. Gerade besonders Ambitionierte bemühten sich um häufige Kontakte zu Einheimischen und nahmen am sozialen Leben in allen Facetten teil, z.B. an den von NGOs angebotenen interkulturellen Treffen, Veranstaltungen, Lern-Cafés, in Sportvereinen und engagierten sich selbst als Ehrenamtliche oder NGO-Mitarbeiter/innen für Personen, die auch geflüchtet sind. Infolge der Ausgangsbeschränkungen und Kontaktverbote fielen diese Möglichkeiten, die deutsche Sprache zu praktizieren und deutschsprachige Kontakte zu pflegen bzw. neue anzuknüpfen, schlagartig weg. Die Expert/inn/ en der NGOs haben in den Interviews auf die negativen Auswirkungen der COVID19-Maßnahmen hingewiesen, indem der Wegfall der Präsenzkurse das Erlernen des Deutschen erheblich beeinträchtigte. Inwieweit andere digitale Lernmöglichkeiten wie die ersatzweise angebotenen Online-Kurse oder Deutschlern-Apps dies ausgleichen konnten, kann auf Basis des vorliegenden Materials nicht treffsicher beurteilt werden.

\section{Good-Practice-Empfehlungen}

- Die vielfältigen Aktivitäten der NGOs zur Kompensation der ausgefallenen Präsenzkurse sind umfassend zu unterstützen. Digitale Lernmodelle und -curricula zum Erlernen der deutschen Sprache wurden seitens der NGOs und Sprachschulen angeboten, ihre langfristige Effizienz bzw. Gleichwertigkeit mit Präsenzkursen kann schwer beurteilt werden. Lernen und die Sprachpraxis in Interaktionen beinhaltet auch eine wichtige soziale Komponente, die digital nicht völlig kompensiert werden kann.

- Auch in der herausfordernden Pandemie sollte die Kontinuierlichkeit des Angebots, aber auch die Möglichkeit der Teilnahme an Deutschkursen gewährleistet sein.

- Die Unterbrechung bzw. der Wegfall angebotener bzw. bereits gestarteter Kurse wurden als Negativfolgen der Pandemie häufig genannt. Aus Vorstudien ist bekannt, dass die Berufstätigkeit von geflüchteten Frauen durchwegs befürwortet und auch ökonomisch begründet wird. Vor allem gebildete Frauen sehen darin auch einen Schritt in Richtung ihres Empowerments und gesellschafticher Teilhabe. Allerdings wurden Frauen schon vor der Pandemie mit dem Problem konfrontiert, dass zu wenige Deutschkurse in Kombination mit Kinderbetreuungsmöglichkeiten existierten. Die nun zusätzlichen Verpflichtungen infolge Homeschoolings, Arbeitslosigkeit der Männer etc. haben die Belastungen für 
Frauen also erheblich verschärft. Umso dringlicher sind frauenspezifische Bildungsangebote und Deutschkurse in Kombination mit Kinderbetreuungsmöglichkeiten.

- Eine Diversifizierung des Deutschkursangebotes nach Aufnahme- und Leistungsfähigkeit, Altersgruppen, Bildungshintergrund und vorhandenen Fremdsprachenkenntnissen ist ein Desiderat im Interesse einer Optimierung des Outputs der Sprachkurse und sollte auch in COVID-Krisenzeiten gewährleistet sein.

- Für das Homeschooling sind neben der Zurverfügungstellung von Gratis-ITEquipment auch weitere Hilfestellungen dringend notwendig, um sozioökonomisch ohnehin benachteiligte Kinder aus den Familien mit Fluchterfahrungen hinsichtlich ihrer Bildungslaufbahnen nicht noch stärker zu benachteiligen. Zusätzlich zur gut frequentierten „Sommerschule“ des Sommers 2020 mit Schwerpunkt auf Vermittlung des Deutschen sind weitere zusätzliche Lernförderprogramme für Kinder mit nichtdeutschen Muttersprachen mittelfristig dringend nötig. Diese könnten ebenfalls von Lehramtsstudierenden unter Anrechenbarkeit für deren Studium durchgeführt werden. Auch sollte das Lehrpersonal davon besonders in Kenntnis gesetzt werden, da dieses die besten Einblicke und Erfahrungswerte aufweist. Lehrer/innen könnten so zu Vermittlungsinstanzen für den weiteren Spracherwerb der Eltern werden (ein Best-Practice-Beispiel ist das in Wien realisierte Projekt „Mama lernt Deutsch“).

- Wir sehen auch die Notwendigkeit professionellen psychologischen Supports bei bildungsbezogenen (Motivations-)Problemen, als Hilfe zum Durchhalten der Mehrfachbelastungen infolge des Homeschoolings, der COVID-19-bedingten Unterbrechungen auf dem Bildungsweg oder wenn sich angestrebte Berufslaufbahnen infolge der ökonomischen Krise als nicht realisierbar herausstellen. Nach ausgesetzten oder unterbrochenen Ausbildungsschienen (AMS, Zweiter Bildungsweg) sollten sich die Organisationen dieser Probleme bewusst annehmen und zusätzliches Coaching entwickeln.

- Nicht nur für Jugendliche mit Fluchterfahrung, sondern auch für Migrant/inn/ en oder Jugendliche aus bildungsfernen Schichten, die sich in keinen Aus-und Weiterbildungsmaßnamen befinden, sollten weitere niederschwellige Angebote geschaffen werden, um mit ihnen die spezifischen Probleme zu thematisieren und an Lösungen zu arbeiten. 


\section{Familiäre Probleme und Konflikte}

Familiäre Probleme wurden in vielerlei Hinsicht virulent, von einfachen Konflikten in der Partnerschaft bedingt durch finanzielle Probleme und Arbeitsplatzverlust, das beengte Zusammenwohnen auf geringen Wohnflächen, die häusliche Präsenz der Kinder durch das Homeschooling und die Sperre von Parks und allen Bereichen der öffentlichen Naherholung in Wien bis zu echten Gewaltproblematiken. Dies führte einerseits zu Depressionen bei den Eltern und Angst bei den Kindern, die sich zudem vor dem Jugendamt fürchteten und selbst bei ernsten Konflikten in der Familie Zweifel hatten, sich kompetenten Personen anzuvertrauen. Auch hier haben die Vereine der Communities, die oftmals mit diesen Konflikten konfrontiert wurden, aber auch die NGOs Erhebliches im Sinne einer Konfliktverminderung und -schlichtung geleistet. Es soll nicht verschwiegen werden, dass auch die patriarchalen Rollenmuster der afghanischen Gesellschaft eine wichtige Rolle spielen. Partnerschaftliche Umgangsformen, wie in Europa üblich, müssen von vielen erst erlernt werden. Nicht nur Frauen, sondern auch Männer haben bezüglich familiärer Probleme die Organisationen der Communities um Hilfe ersucht. Unser empirisches Material deutet auf eine Zunahme familiärer und partnerschaftlicher Konflikte hin, diese ist allerdings nicht quantifizierbar und einer Vielfalt an Auslösungsfaktoren zuweisbar. Konkrete Statistiken bezogen auf die Haushalte fehlen hierzu, jedenfalls sind gemäß den Aussagen von Angehörigen der afghanischen Communities die Scheidungsraten merklich angestiegen. Bereits seit Jahren werden steigende Scheidungszahlen nach der Flucht in syrischen Communities thematisiert. Es ist anzunehmen, dass sich diese Entwicklung während der COVIDKrise verschärft hat. Offizielle Zahlen gibt es hierzu derzeit nicht. Viele Problemkonstellationen sind überdies komplex miteinander verzahnt. So konnte die Angst vor dem Alleinsein eine Art Flucht ins Zusammenwohnen bedingen, was jedoch aufgrund der erzwungenen langfristigen Nähe und dem Mangel an Rückzugsmöglichkeiten erst recht wieder zu Partnerschaftsproblemen führte. Wie auch in der österreichischen Mehrheitsgesellschaft tragen Frauen hierbei einen Großteil der zusätzlich zu bewältigenden Aufgaben und es bedarf mehr als nur eines gesellschaftlichen Bewusstseins dafür.

\section{Good-Practice-Empfehlungen}

- Der Ausbau von existierenden professionellen Beratungsinfrastrukturen zur Bewältigung familiärer Probleme und Gewalt in Ehe und Familie bedarf dringend weiterer Unterstützung. Dies vor allem auch angesichts des aktuellen Lockdowns und etwaiger weiterer Lockdowns, durch die von einer weiteren Zunahme dieser Probleme auszugehen ist. Frauen benötigen hierin mehr ökonomische und psychosoziale Unterstützung. Bei weiteren Maßnahmen zur Pandemiebewältigung müssen die geschlechtsspezifischen Folgewirkungen besser antizipiert und zusätzliche Angebote geschaffen werden. 
- Patriarchale Strukturen verstärken die Problematik, ihnen ist vor allem mit Männerberatung sowie Gruppen- und Einzeltherapien entgegenzutreten, die etablierte Rollenschemata aufbrechen und verändern. Dass dies nicht über Nacht erfolgen, sondern längere Zeithorizonte erfordern wird, muss bewusst sein.

- Oft kommt Unterstützung bei familiären Schwierigkeiten aus informellen Netzwerken innerhalb von Vereinen, Communities oder der erweiterten Familie. Um schwerwiegende Krisen zu vermeiden, müssen auch informelle Netzwerke gestärkt werden. Dies könnte durch regelmäßige Rechtsberatungen von Schlüsselpersonen in Communities geschehen sowie durch eine klare Kommunikation von Entlastungsanbeboten (wie etwa Kinderbetreuung etc.) in den Muttersprachen innerhalb der Communities und Vereinen. Zudem muss auch während geltender Kontaktbeschränkungen die Möglichkeit gegeben sein, informelle Hilfe in Krisensituationen in Anspruch zu nehmen. Um rechtliche Unklarheiten zu vermeiden, wäre hier eine in verschiedene Zielsprachen übersetzte Guideline eine wichtige Orientierungshilfe für Betroffene, Helfende und Exekutive. 


\section{NGOS UND VEREINE DER COMMUNITIES SOWIE UNTER- STÜTZUNGSANGEBOTE}

Im Rahmen der Online-Befragung wurde auch erhoben, an wen man sich wandte, wenn Unterstützung bei pandemiebedingten Problemen benötigt wurde. Ein Fünftel aller Befragten hat sich um Unterstützung an österreichische Freund/inn/e/n oder Bekannte gewendet, 17\% haben Hilfe bei Freund/inn/en aus der eigenen Community erbeten und 13\% haben institutionalisierte Hilfe bei Ämtern, Behörden oder Kammern gesucht. Weniger wichtig waren mit $8 \%$ die NGOs sowie mit gleichen Prozentanteilen afghanische bzw. syrische Vereine oder Organisationen anderer Herkunftsgruppen. Ehrenamtliche Helfer/innen wurden etwas seltener um Hilfe ersucht und am wenigsten wandten sich die Befragten an religiöse Vereine oder Moscheen.

Der Aspekt der Relevanz der NGOs wurde bereits in einigen vorangehenden Punkten angesprochen, denn die Coronakrise hat das Unterstützungsangebot aller NGOs im Kontext der Betreuung Geflüchteter auf vielfältige Weise verändert. Die markanteste Wandlung war die Umstellung der Kontaktmöglichkeiten auf Online- bzw. telefonische Kontakte. 28\% der Befragten gaben diesbezüglich jedoch an, dass damit auch die Unterstützungsangebote und der Zugang zu selbigen komplizierter geworden sind. Für 14\% war damit eine Verminderung an Unterstützung verbunden und immerhin ein Zehntel gab an, dass vorher vorhandene Unterstützung sogar gänzlich wegegefallen sei. Zusätzliche Angebote haben nur 6\% registriert. Untersucht wurde diesbezüglich nicht in welcher Form diese Unterstützungsleistungen erfolgten (in materieller oder ideeller Form). Bei letzterer könnte diese nicht als eine Unterstützungsleistung gesehen worden sein, sondern eher als ein Teil der sozialen Beziehungen, die sich die Menschen in den letzten Jahren aufgebaut hatten.

Im Vergleich der Herkunftsgruppen wurde von fast der Hälfte der syrischen Befragten die Beschränkung der Kontakte zu NGOs auf digitale Medien bzw. Telefon erwähnt, bei den Afghan/inn/en war dieser Anteil beträchtlich geringer. Syrische Respondent/inn/en haben auch viel öfter angegeben, dass das Unterstützungsangebot der NGOs komplizierter wurde. Unter den Afghan/inn/en waren weniger dieser Ansicht. Dafür haben diese viel öfter erfahren müssen, dass die Unterstützung reduziert wurde oder sogar ganz weggefallen ist. Etwas mehr syrische Befragte als afghanische erwähnten zusätzliche Angebote seitens der NGOs. Hierbei muss berücksichtigt werden, dass das Angebot an arabischsprachigen Vereinen oder Organisationen in Wien (d.h. nicht alleine aufgrund der syrischen Herkunft/Zugehörigkeit) numerisch viel größer und differenzierter ist als jenes für Afghan/inn/en. Auch haben manche Vereinigungen eine lange Tradition, sprechen nicht alleine eine ethnonationale oder religiöse Gruppe an, sondern sind breiter aufgestellt. Deren Netzwerke waren daher während der Pandemie leichter ausbaubar.

Bei der Frage nach den vor allem unterstützten Personengruppen war es besonders die eigene Familie, die von den Befragten am meisten Unterstützung erfahren hat (61\% 
sehr oft bzw. oft). Viel seltener wurden Angehörige aus der eigenen Herkunftscommunity, die aber nicht der Familie angehörten, unterstützt. Aber immerhin ein Viertel der Befragten tat dies sehr oft bzw. oft, hingegen 58\% nur selten. Personen mit anderem Migrationshintergrund wurden wesentlich seltener mit Support bedacht, denn $47 \%$ taten dies gar nicht bzw. 38\% selten. Noch geringer waren die Unterstützungsleistungen für Angehörige der eigenen Glaubensgemeinschaft, die nur von 10\% der Befragten sehr oft/ oft Unterstützung erhielten. Ob und inwieweit Transformationen in den eigentlich recht bedeutsamen religionsspezifischen Vereinen bereits in den letzten Jahren erfolgt sind oder sich durch die Pandemie ergeben haben, bedarf weiterer Untersuchungen.

In den Expert/inn/en/interviews wurden die vielfältigen Veränderungen der Unterstützungsangebote beschrieben, wobei die grundlegendsten Änderungen im Wegfall persönlicher Kontakte, der kompletten Umstellung auf Online-Angebote und somit Schwerpunktverlagerungen des gesamten Angebots kam. Während die NGOs und deren Mitarbeiter/innen nach unmittelbaren Anfangsschwierigkeiten meist recht gut mit der geänderten Situation umzugehen lernten, kam nur ein Teil der Klient/inn/en der NGOs mit diesen Veränderungen wirklich gut zurecht. Auch seitens der Ehrenamtlichen kam es zu massiven Einschnitten. Sie mussten ihr Engagement häufig völlig ruhendstellen bzw. ebenfalls auf Handy bzw. online umstellen. Für die NGOs gingen damit auch wichtige Personalressourcen verloren, die auch nach Aufhebung des Lockdowns keineswegs mehr in vollem Umfang kompensiert werden konnten. Des Weiteren muss auch hier die Überlastung des Personals in NGOs und GOs berücksichtigt werden, da viele auch zusätzliche Betreuungsleistungen übernommen haben.

Im Kontext des für das Projekt gewählten methodischen Ansatzes der Community Based Participatory Research (CBPR) spielten die Vereine der Communities eine wichtige Rolle (RoBERTs 2013). Nicht nur in den syrischen und afghanischen Communities haben sich eigene Initiativen entwickelt, die helfen, sich in Österreich zurechtzufinden, sondern dies gilt für viele Herkunftsgruppen. Im Falle der Syrer/innen existiert eine Internetgruppe, die mit ihren mehr als 56.000 Mitgliedern inzwischen weit mehr Personen umfasst, als neuangekommene Syrer/innen, für welche sie ursprünglich ins Leben gerufen worden war. In diesen Gruppen werden Informationen ausgetauscht, Probleme und Herausforderungen in Österreich diskutiert und gegenseitige Unterstützung (z.B. Übersetzungen, Informationen zu Ansprechpartner/inne/n) ausgetauscht. Die Administrator/inn/en fungieren dabei als Lots/inn/en, um Falschinformationen zu verhindern. Dies ist oft mit einem hohen Zeitaufwand verbunden. Die Bedeutung dieser Gruppen vor allem auch in der herausfordernden Pandemie sowie der Einsatz bestimmter Administrator/inn/en und besonders aktiver Gruppenmitglieder ist bisher kaum sichtbar gemacht worden. Zudem gibt es auch Offline-Initiativen, in welchen Familien einander gegenseitig beim Erlernen der Sprache oder der Bewältigung von Alltagsproblemen in Pandemiezeiten helfen (vgl. Six-Hohenbalken 2020). 


\section{Good-Practice-Empfehlungen}

- Viele Befragte stammen aus Herkunftskontexten, in denen der Face-to-FaceKontakt noch eine viel wichtigere Rolle spielt als in Europa. Es ist grundsätzlich sehr positiv zu bewerten, dass es den NGOs gelungen ist, ihr Angebot rasch umzustellen, allerdings darf nicht außer Acht gelassen werden, dass damit nicht mehr alle Klient/inn/en erreicht werden konnten. Auch mussten schichtspezifisch (beispielsweise nach Alter, Bildungsstand) unterschiedliche Internetmedien genutzt werden. Die Kompensation der dadurch entstandenen Defizite nach der Coronakrise muss ein wichtiges Ziel sein.

- Die Online-Befragung hat ergeben, dass es vor allem Freunde und Bekannte waren, an die man sich um Hilfe und Unterstützung wandte, und weniger die NGOs der Flüchtlingsbetreuung. Hier dürfte also trotz aller Bemühungen der Organisationen seitens der Zielgruppen noch immer eine gewisse Barriere vorhanden sein, die zahlreichen vorhandenen Hilfestellungen auch anzunehmen. Des Weiteren kommt zum Vorschein, dass es bereits vor der Pandemie Zusammenschlüsse von Familien gegeben hat, die einander gegenseitig in Notfällen auch finanziell unterstützt haben. Während der Pandemie sind auch solche Vernetzungen besonders bedeutend geworden.

- Expert/inn/en plädierten vor allem in Zeiten der Krise und Pandemie zur Vermeidung von Ineffizienzen für eine Verbesserung organisatorischer Abläufe sowie für ein Mehr an Koordination innerhalb und zwischen den involvierten Organisationen und Institutionen. Damit in einem Konnex steht auch die Empfehlung, bestehende Kommunikationsdefizite zu minimieren und auf institutioneller Ebene möglichst eng zusammenzuarbeiten.

- Dringend notwendig ist die Würdigung und Sichtbarmachung des zivilgesellschaftlichen Engagements, das von diasporischen Gemeinschaften geleistet wird. Gerade diasporische Gruppen und Institutionen der Communities leisten wesentliche Beiträge in der Unterstützung von besonders vulnerablen Gruppen, die nicht in die staatlichen Integrationsschienen aufgenommen wurden (Beispiele gibt es in Deutschland bezüglich Sprachelernen und Arbeitssuche). Solche Bereiche müssen ausgemacht und auch besonders unterstützt werden, u.a. in Form materieller Leistungen. Wenn es gruppenzentrierte Unterstützungen geben soll, dann braucht es ein Mindestmaß an Standards, die dabei eingehalten werden müssen.

- Es wird mehr aktive und finanzielle Hilfe für Beratungseinrichtungen, Institutionen der Flüchtlingshilfe sowie die Organisationen der ethnischen Communities empfohlen. Anerkennung von und Unterstützung für diese Initiativen ist dringend nötig. Die Unterstützungen diasporischer Gemeinschaften in ihren Integrationsmaßnahmen müssen mit besonderer Umsicht und speziellem Wissen erfolgen, da daraus auch kompetitive Beziehungen zwischen einzelnen Organi- 
sationen resultieren können. Denkbar wäre es auch, Administrator/inn/en mittels spezieller Schulungen zu unterstützen. Auch hier wäre es denkbar, diese Anstrengungen mehr anzuerkennen und eventuell mit Informationen zu kostenlos nutzbaren Räumlichkeiten oder Förderungen seitens der Gebietskörperschaften zu unterstützen.

- Auch Expert/inn/en aus den ethnischen Communities und vor allem deren Organisationen sollten in Beratungs- und Organisationsprozesse zu pandemiebezogenen Maßnahmen involviert werden, um aus ihrem Kontextwissen entsprechenden Mehrwert zu ziehen.

- Maßnahmen sollen keinesfalls als ,,von oben “ aufoktroyiert empfunden werden, denn dieses Gefühl verringert erheblich die Bereitschaft zu deren Akzeptanz.

- Die Förderung von Gatekeepers in Form eines bewussten Diversitätsmanagements beispielsweise im Ausbildungsbereich (Sozialarbeit, Gemeinwesenarbeit) ermöglicht auf lange Sicht eine dialogische Kooperation und die Einbindung jener Integrationskompetenzen, die Mitglieder diasporischer Gemeinschaften mitbringen.

- Es muss ein Bewusstsein dafür geschaffen werden, dass die Unterstützungen von/ durch diasporische Gemeinschaften auch ein hohes Maß an Vulnerabilität für die diversen Akteur/inn/e/n impliziert. Diese bzw. deren Eltern haben vielleicht selbst Fluchtbiographien aufzuweisen und sind gerade auch deshalb besonders exponiert und bedürfen besonderer Unterstützung (Supervision etc.).

- Diasporische Organisationen oder Institutionen können ihrerseits auch die Kontakte mit der Residenzgesellschaft ermöglichen oder erleichtern. Die Einbindung und Förderung dieser Institutionen soll daher aufjeden Fall dialogzentriert und integrationsfokussierend sein. Im Ausbau dieser Kompetenzen mit besonderer Berücksichtigung der Kommunikation und Vermittlung sowie der Bearbeitung der Intersektionalität von Problemlagen (medizinisch, rechtlich, bildungspolitisch) können für die gesamte Gesellschaft neue Modelle konzipiert und entwickelt werden. 


\section{ASYlWERber/INNEN UND ASYlQuarTiERe}

Spezifische Probleme stellten sich bei jenen Personen, die noch Asylwerber/ innenstatus innehatten, sich in der Grundversorgung befanden und somit noch in betreuten und oft überfüllten Asylquartieren lebten. Es handelte sich hierbei zwar um eine sehr kleine Gruppe von Respondent/inn/en, aber mit besonderen Problemen. Hier war die Einhaltung der Coronamaßnahmen besonders schwierig, vor allem wenn in größeren Familien einzelne Personen positiv getestet wurden oder wenn in den jeweiligen Quartieren viele Coronaverdachtsfälle in kurzen Intervallen auftauchten. Die Trägerorganisationen befanden sich hier oft in schwierigen Situationen, da primär den Anordnungen der Gesundheitsbehörden Rechnung getragen werden musste. Hier wurde sowohl seitens der Befragten aus den Communities als auch der Expert/inn/en aus den Trägerorganisationen Kritik geäußert. Die an den Bund errichtete naheliegende Forderung bestand darin, Großquartiere möglichst aufzulösen und kleinere Einheiten zu schaffen. Dies wäre natürlich durchwegs die vernünftigere Lösung gewesen, aber man konnte keine Kapazitäten aus dem Nichts hochfahren, da die Basisstrukturen dafür fehlten. Expert/inn/en von NGOs berichteten weiters von falschen Informationen, die an die Asylwerber/innen weitergegeben wurden, von strikten Verboten, die eigentlich nicht maßnahmenkonform gewesen waren und von starken psychischen Belastungen infolge des Zusammenlebens auf engem Raum mit sehr wenig Selbstbestimmung.

\section{Good-Practice-Empfehlungen}

- Im Rahmen der Grundversorgung wäre das primäre Desiderat - nicht nur während der COVID-19-Krise - kleinere Unterbringungseinheiten anzustreben, wie dies in einigen Bundesländern ja bereits erfolgreich umgesetzt werden konnte. Damit einher ginge nicht nur eine Reduktion der Infektionsrisiken generell, sondern auch eine Verminderung der psychischen Belastungen für die in Grundversorgung befindlichen Personen und die Stärkung der Agency der Betroffenen.

- Hinsichtlich der Informationsdiffusion ist stärker auf die Verbreitung ausschließlich maßnahmenkonformer Informationen an die in Grundversorgung befindlichen Personen zu achten. 